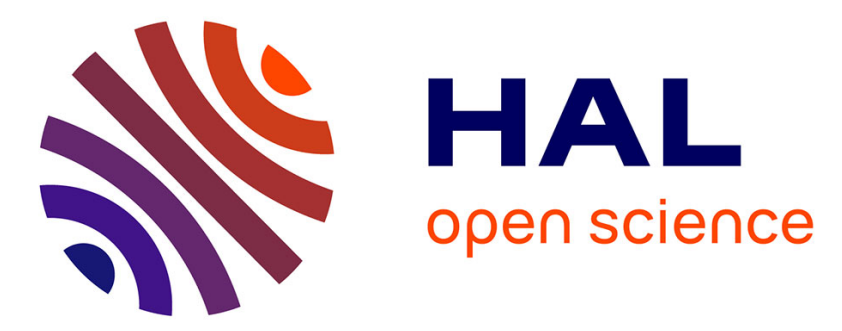

\title{
Numerical study of a rotor/stator interaction case experimentally simulated with an industrial compressor
}

Alain Batailly, Mathias Legrand, Millecamps Antoine, François Garcin

\section{To cite this version:}

Alain Batailly, Mathias Legrand, Millecamps Antoine, François Garcin. Numerical study of a rotor/stator interaction case experimentally simulated with an industrial compressor. ASME Turbo Expo 2012 Turbine Technical Conference and Exposition, Jun 2012, Copenhaguen, Denmark. pp.10531062, 10.1115/GT2012-68173 . hal-00714538

\section{HAL Id: hal-00714538 \\ https://hal.science/hal-00714538}

Submitted on 4 Jul 2012

HAL is a multi-disciplinary open access archive for the deposit and dissemination of scientific research documents, whether they are published or not. The documents may come from teaching and research institutions in France or abroad, or from public or private research centers.
L'archive ouverte pluridisciplinaire HAL, est destinée au dépôt et à la diffusion de documents scientifiques de niveau recherche, publiés ou non, émanant des établissements d'enseignement et de recherche français ou étrangers, des laboratoires publics ou privés.

\section{(c)(1)}

Distributed under a Creative Commons Attribution| 4.0 International License 


\title{
Numerical study of a rotor/stator interaction case experimentally simulated with an industrial compressor
}

A. Batailly, Laboratoire de dynamique des structures et vibrations, université McGill, Québec M. Legrand, Laboratoire de dynamique des structures et vibrations, université McGill, Québec A. Millecamps, Snecma, site de Villaroche, France F. Garcin, Snecma, site de Villaroche, France

\begin{abstract}
Higher aircraft energy efficiency may be achieved by minimizing the clearance between the rotating blade tips and respective surrounding casing. A common technical solution consists in the implementation of an abradable liner which improves both the operational safety and the efficiency of modern turbomachines. Recently, unexpected abradable wear removal mechanisms were observed in experimental set-ups and during maintenance procedures. The present study introduces a numerical strategy capable to address this occurrence.

After focusing on the analysis of the experimental results, the good agreement between experimental observations and numerical results is illustrated in terms of critical stress levels within the blade as well as final wear profiles of the abradable liner. New blade designs are also explored in order to assess the impact of blade design on the outbreak of the interaction phenomenon. The prevalence of three dominant parameters in the interaction onset is shown: (1) blade design, (2) abradable material mechanical properties and (3) the need for a global distortion of the casing to synchronize blade-tip/abradable coating contacts.
\end{abstract}

\section{Introduction}

From a structural point of view, experiments conducted in vacuum environments [1] showed that rotor/stator interactions sometimes lead to unacceptable damages to both the rotating and static mechanical components (see Fig. 1). Three distinct phenomena have been identified:

1. the traveling wave speed coincidence (also termed as modal interaction) [2] which may be described as a geometric matching between the vibration modes of both the rotating structure (bladed disk or single blade) and the surrounding casing [3]. Amplitudes of vibrations of both structures are sustained (or increased) over time due to direct structural contacts.

2. the rubbing phenomenon is characterized by significant wear on the casing or/and on the blade-tip [4]. In essence, this phenomenon involves a single blade and may be localized on the casing.

3. the whirl/whip phenomena [5] are unstable motions arising due to precessional orbits in the rotor-bearing system.

The distinction between the aforementioned phenomena is arduous since they are usually coupled. In the present study, it is assumed that the experiments involve both modal interaction and rubbing. Whirl/whip phenomena are not considered because both disk dynamics and shaft vibration were shown to be negligible during the experiment.

At least two manufacturers observed rubbing [6] or blade cracks [1] after blade-tip/abradable coating contacts over the last five years. Many experimental work may be found regarding the static characterization of this material $[7,8]$ but its dynamic characterization is still under investigation.

The finite element method coupled with an optimization procedure was used in [9] for the simulation of the HR15Y hardness test on abradable material. It was shown that a bilinear plastic law could accurately represent the mechanical behavior of abradable material. Consistently, recent numerical developments focused on the modeling of abradable coating through mono-dimensional plastic elements [10] in the context of full 3D blade-tip/abradable coating simulation. Recent applications of this strategy [11] proved promising results in good agreement with the experimental data.

The first section exploits the industrial experiment and respective results given in [1]. The numerical strategy proposed in [10] is summarized and the comparison with the existing analysis is presented. Finally, sensitivity of the interaction phenomenon and possible related divergence to new blade designs is explored. 


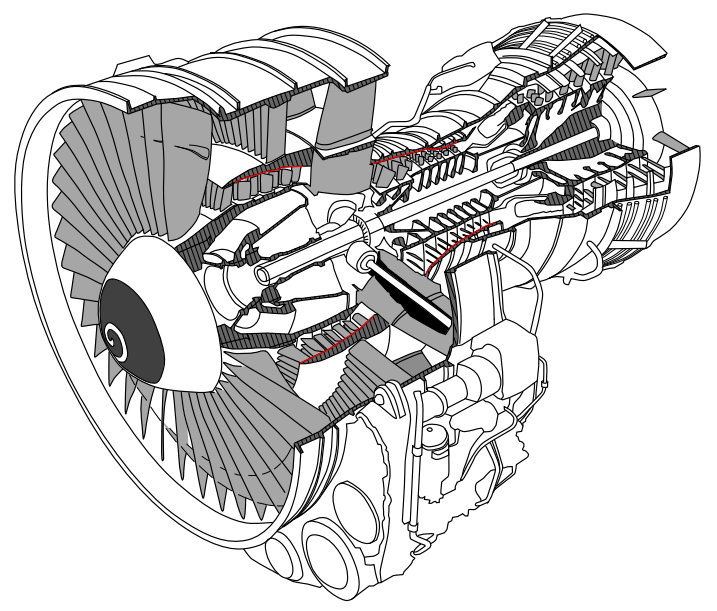

Figure 1 - Cut-view of an aircraft engine with sensitive contact areas $(-)$.

Note 1: In this paper, experimental data from [1] are plotted in red while numerical results are plotted in blue.

Note 2: Results are normalized for the sake of confidentiality. Frequencies are normalized with respect to the first eigenfrequency of the blade at rest $f_{1}$ and time is normalized with respect to one revolution of the blade at the interaction speed: $\mathrm{T}_{\Omega \text {,int }}=1 / f_{\Omega \text {,int }}$ where $f_{\Omega \text {,int }}$ is the rotational frequency for which the interaction phenomenon is experimentally observed.

\section{Industrial experiments}

\section{Set-up}

The experimental bench used for the blade-tip/abradable coating application is partially depicted in Fig. 2 and is detailed in [1]. A full scale experimental multi-stage compressor of an aircraft engine is driven by an electric motor in a vacuum chamber in order to avoid possibly disturbing noise sources like temperature and aerodynamics loadings. The last low-pressure compressor stage only has blades, among which one is slightly longer than its counterparts and pictured in gray in Fig. 2. The interaction phenomenon involves the longer blade, which is instrumented with a strain gage $(G)$ located on its suction side. This strain gauge measures the stresses in the middle of the blade along the radial direction $\sigma_{\mathrm{rr}}$.

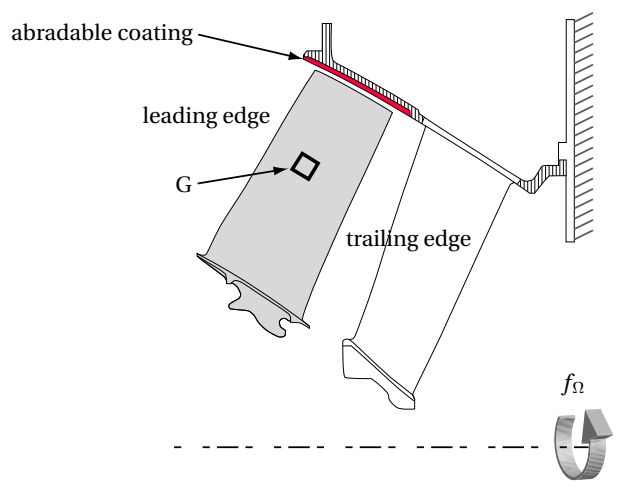

Figure 2 - Experimental set-up.

The last stage of the compressor is a downstream guide vane screwed on a heavy frame and the blade pictured in white in Fig. 2 is attached to the casing. An abradable coating facing the blade of interest is sprayed on the casing.

\section{Experimental scenario}

The Campbell diagram of the longer blade ${ }^{1}$ of interest is given in Fig. 3. Because of centrifugal stiffening, the first eigenfrequency $f_{1}$ of the blade increases with the rotational frequency $f_{\Omega}$. Beside of $f_{1}$,

\footnotetext{
${ }^{1}$ This diagram is obtained numerically with a full $3 \mathrm{D}$ finite element model of the blade.
} 
six engine orders ${ }^{2}$ are drawn for $k=3,4,5,6,7$ and 8.

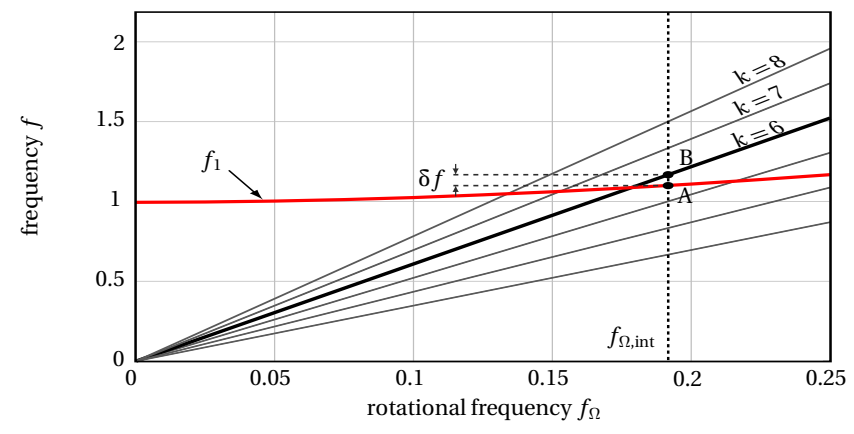

Figure 3 - Campbell diagram of the blade of interest.

The chosen experimental rotational frequency is $f_{1}=5.5 f_{\Omega}$. Initially rotating at low frequency, the compressor undergoes a high acceleration between $t=110 \mathrm{~s}$ and $t=135 \mathrm{~s}$ to reach rotational frequency $f_{\Omega}=f_{\Omega \text {,int }}$ as shown in Fig. 4 on which the time interval of the interaction $\Delta t$ is underlined. Blade rubbing against the casing is achieved through centrifugal load.

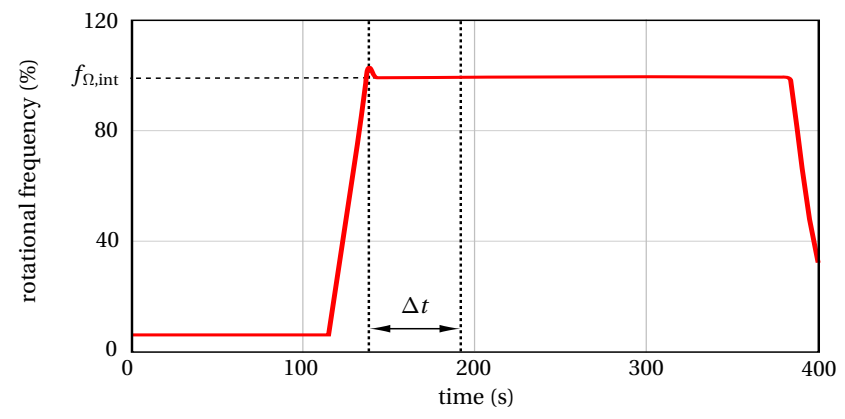

Figure 4 - Evolution of the rotational speed during the experiment.

\section{Results}

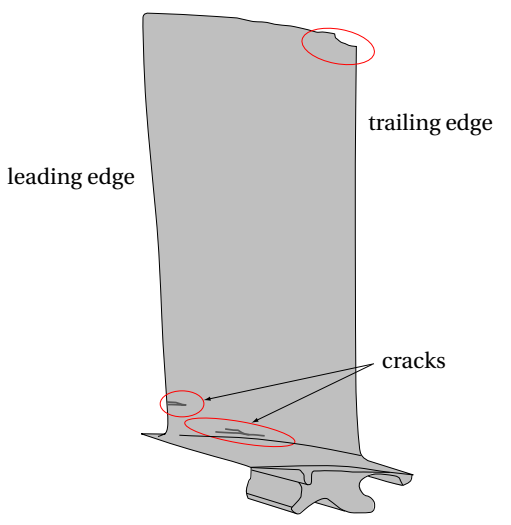

Figure 5 - Schematic representation of the blade after the test.

At the end of the test run, cracks in the blade were noticed and both its dovetail and trailing edges were damaged similarly to what is pictured in Fig. 5. While the blade presented color modifications due to a very local overheating at its tip, its overall temperature did not significantly vary during the experiment and it is suggested that friction related thermal effects did not affect the dynamic characteristics of the blade.

Several sudden changes were also observed. Five phases may be distinguished in the stress levels acting in the middle of the blade once the targeted rotational frequency $f_{\Omega \text {,int }}$ is reached (around $t=138 \mathrm{~s})$. The five phases of the test are displayed in Fig. 6(a):

\footnotetext{
${ }^{2}$ An engine order is defined by the linear relation $f=k f_{\Omega}, k$ being an integer.
} 


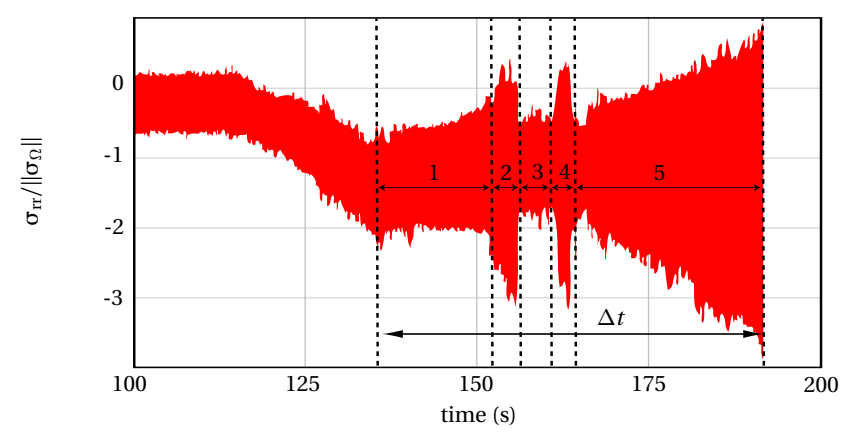

(a) Time domain (blade failure at $t=190 \mathrm{~s}$ ).

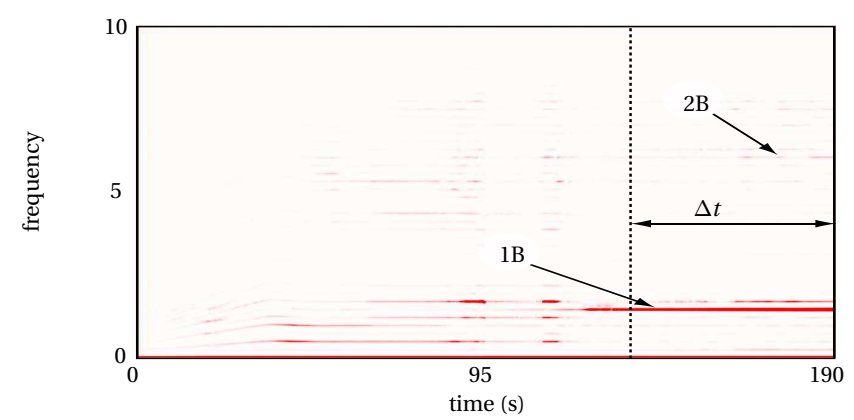

(b) Spectrogram (1B: first bending mode).

Figure 6 - Stress in the middle of the blade during the experiment.

phase 1 from $t=138 \mathbf{s}$ to $t=155 \mathbf{s}$ Stress in Fig. 7(a) in the blade is essentially periodic over one revolution of the blade, its mean value $\sigma_{r r, m}$ - which is non zero due to centrifugal stiffening is $\sigma_{r r, m}=-1.18$. From Fig. $7(\mathrm{~b})$, response features dominant even harmonics thus suggesting evenly distributed contact occurrences per blade revolution. More precisely, the dominance of

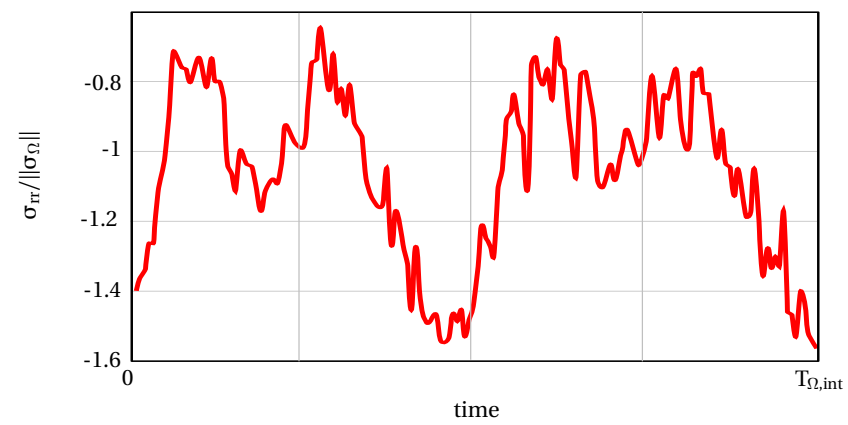

(a) Blade stress in the middle of the blade

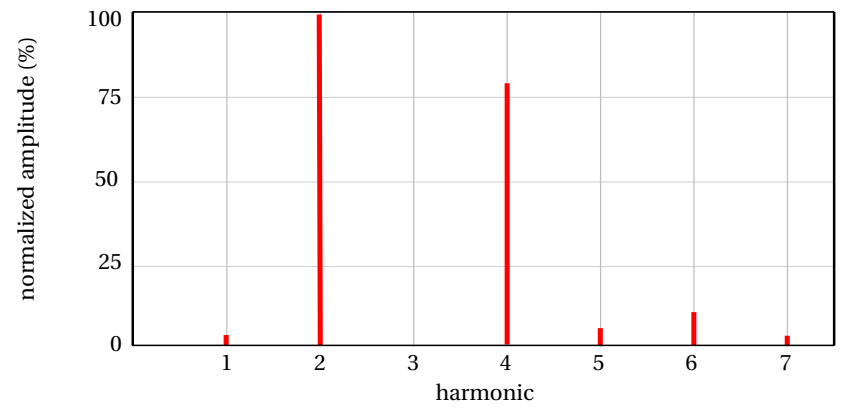

(b) Harmonic content of the blade stress signal.

Figure 7 - Experimental results [1]: blade stress in the middle of the blade during the first phase and corresponding spectrum.

harmonic 2 evokes a possible ovalization of the casing.

phase 2 from $t=155 \mathbf{s}$ to $t=158 \mathrm{~s}$ a complex unstable blade stress behavior is observed with a very 
sudden and brief increase of the stress level within the blade.

phase 3 from $t=158 \mathrm{~s}$ to $t=162 \mathrm{~s}$ the stress level in the blade is very low (lower than during the first phase)

phase 4 from $t=162 \mathrm{~s}$ to $t=166 \mathrm{~s}$ similar observations to those about phase 2 are made. Stress level in the blade increases very suddenly.

phase 5 from $t=166 \mathbf{s}$ to $t=190 \mathrm{~s}$ the stress level of the blade increases slowly until the blade is damaged at $t=190 \mathrm{~s}$ and signal is lost. Stress in the middle of the blade is pictured in Fig. 8(a). Its respective harmonic content plotted in Fig. 8(b) emphasizes a predominant sixth harmonic.

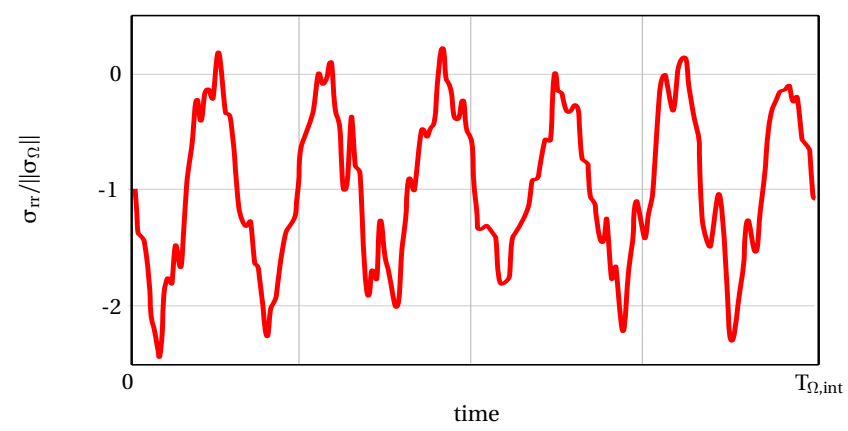

(a) Blade stress in the middle of the blade

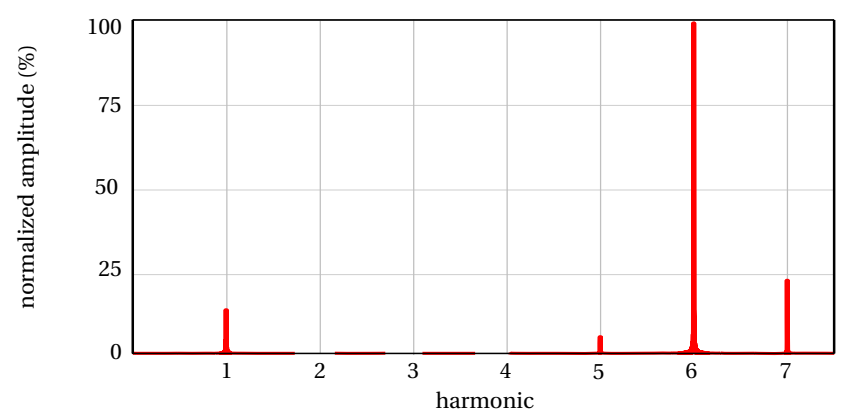

(b) Harmonic content.

Figure 8 - Experimental results [1]: blade stress in the middle of the blade after $190 \mathrm{~s}$ and corresponding spectrum.

Overall, from the spectrogram pictured in Fig. 6(b), when interaction starts, a dominant first bending mode is involved. The wear levels of the abradable coating on the casing are measured both on the trailing edge and the leading edge. The two wear profiles are plotted in Fig. 9.

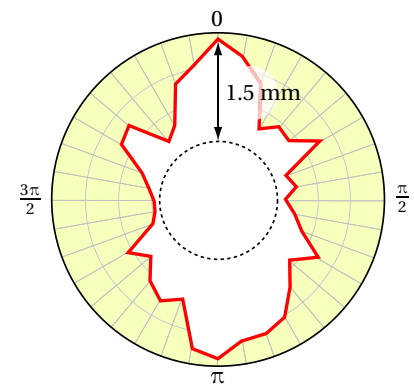

(a) leading edge

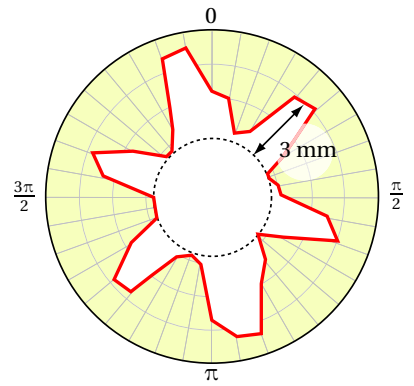

(b) trailing edge

Figure 9 - Wear of the abradable coating observed experimentally [1].

While two wear lobes are measured at the leading edge, six wear lobes are measured at the trailing edge (Fig. 9(b)) thus exhibiting a complex vibration pattern of the blade tip. In addition, the wear level on the trailing edge is significantly higher than the one on the leading edge which is consistent with the predominance of the first bending mode of the blade during the interaction, as underlined in 
Fig. 9. Indeed, a modal analysis of the blade ${ }^{3}$ reveals that the maximum radial displacement of the trailing edge is about three times the maximum radial displacement of the leading edge for the first bending mode.

\section{Solution method}

This section aims at presenting the numerical strategy used for the simulation of the experiment detailed in the first section of the paper. Most of those developments are only briefly recalled and more information may be found in [10] regarding abradable coating modeling, reduced order model taking into account centrifugal effects as well as the contact algorithm.

The blade of interest is pictured in Fig. 10. The industrial 3D finite element model is made of quadratic tetrahedron elements and contains about 25,000 nodes. The two finite elements used for stress analysis - element 1982 in the middle of the blade and element 6455 at the root of the leading edge - as well as the boundary conditions are depicted in Fig. 10. More details regarding the definition of boundary conditions may be found in [10].

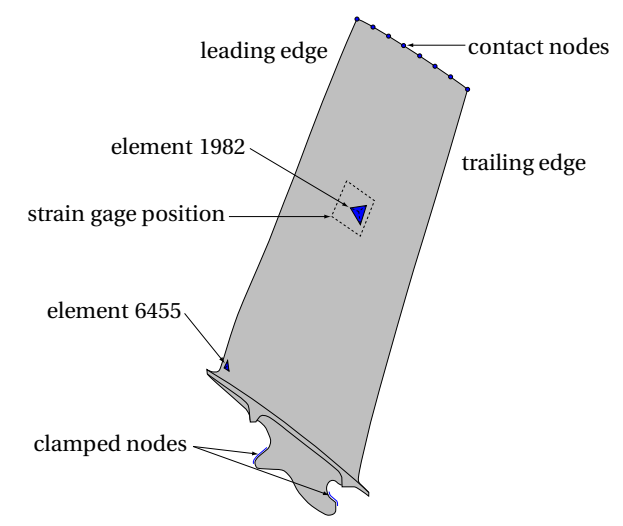

Figure 10 - Blade finite element model.

Equations of motion are derived within the small perturbation framework and the reference frame is attached to the rotating bladed disk. The shaft supporting the bladed disk is perfectly rigid, thus the axis of rotation is fixed. Centrifugal stiffening and its geometrical effect on the blade-tip/casing clearance are accounted for.

The main approximations or differences between the experiment and the numerical simulation are:

1. the rotational velocity of the blade is constant in the numerical simulation as opposed to the experimental set-up.

2. contact between the blade and the casing is initiated with an ovalization of the casing consistently with previous industrial observations when a thermal load is applied on the casing. This ovalization does not account for manufacturing imperfections. As a result, the maximum radial displacement is $d_{\max }=2 c_{0}$ where $c_{0}$ is the blade-tip/casing clearance at rest with a perfectly circular casing. The ovalization is applied following an exponential evolution evol $(t)$ pictured in Fig. 11:

$$
\operatorname{evol}(t)=1-e^{\alpha t}
$$

3. as experimentally observed, the casing is not sensitive to the interaction and is modeled as a rigid mathematical surface over which is deposited the abradable coating.

4. the abradable coating is modeled through a plastic law detailed in [10]. This law involves three mechanical parameters: the Young's modulus E, the plastic modulus K and the yield strength $\sigma_{Y}$. Industrial experience and previous numerical simulations allow for an accurate calibration of these three parameters.

5. in the experiment only the longer blade was involved in the interaction: this is reflected in the developed numerical strategy by modeling a simple blade clamped on its foot.

\footnotetext{
${ }^{3}$ This modal analysis is not detailed in the present paper for the sake of brevity.
} 
6. finally, in order to minimize computation effort, a component mode synthesis method compatible with the contact algorithm is used. This method accounts for centrifugal effects directly within the reduced order model [10]. Its compatibility with the contact algorithm we use has been previously studied in [3] where its advantages in the context of contact simulations with reduced-order models with respect to other component mode synthesis methods were also highlighted. Eight contact nodes - depicted in Fig. 10 - are defined along the blade tip in order to manage contact from the leading edge to the trailing edge.

\section{Interaction simulatioN}

Numerical simulations are carried out around the experimental rotational frequency $f_{\Omega}=f_{\Omega \text {,int }}$. An interaction phenomenon arises for $f_{\Omega \text {,int }}^{*} \simeq f_{\Omega \text {,int }}+5 \%$ and the associated period is $\mathrm{T}_{\Omega \text {,int }}^{*}$. The difference between numerical and experimental interaction rotational frequencies is assumed to be related with the numerical assumptions aforementioned.

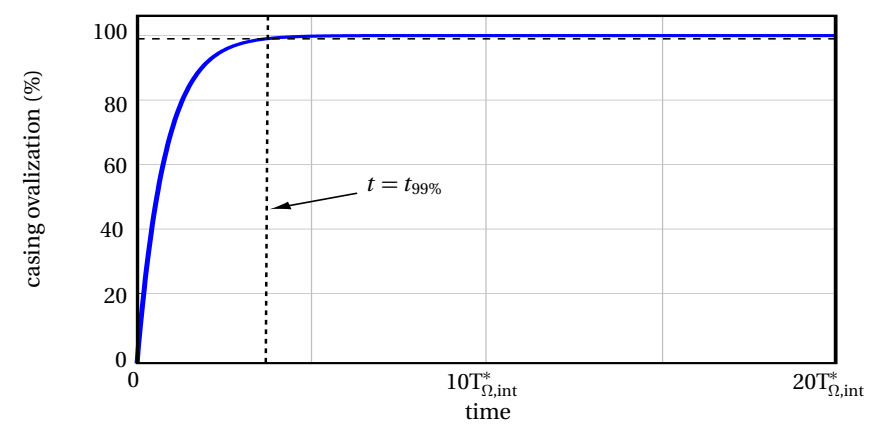

Figure 11 - Ovalization of the casing.

The simulation time corresponds to twenty revolutions of the blade: $t_{\mathrm{sim}} \simeq 20 \mathrm{~T}_{\Omega \text {,int }}^{*}$. This time is shorter than the total duration of the experiment but is comparable with the duration of each interaction phase experimentally observed. Accordingly, it is expected that the simulation time is long enough to catch the ignition of the interaction phenomenon.

The ovalization amplitude with respect to time is pictured in Fig. 11. The time for which the maximal radial displacement reaches $0.99 d_{\max }$ is denoted $t_{99 \%}$. Considering Eq. 1 , it comes:

$$
t_{99 \%}=\frac{\ln (0.01)}{\alpha}
$$

It has been numerically verified that the interaction results presented in the following do not depend on $\alpha$.

\section{General observations}

The tangential displacement on the trailing edge is depicted in Fig. 12. One may observe two distinct phases: (1) for $t \leq t_{99 \%}$ the amplitude of the displacement increases quickly (slope $s_{1}$ in Fig. 12) due to the ovalization of the casing and (2) for $t \geq t_{99 \%}$ the amplitude grows almost linearly (slope $s_{2}$ in Fig. 12) while the casing has reached its final shape.

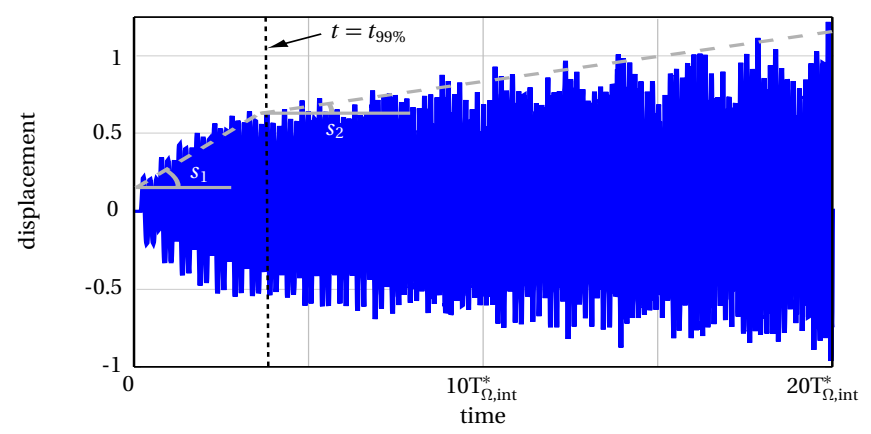

Figure 12 - Tangential displacement of the contact node on the trailing edge.

Periods of time $t \in\left[0 ; t_{99 \%}\right]$ and $t>t_{99 \%}$ will be respectively referred to as phase 1 and phase 2 in the sequel. 


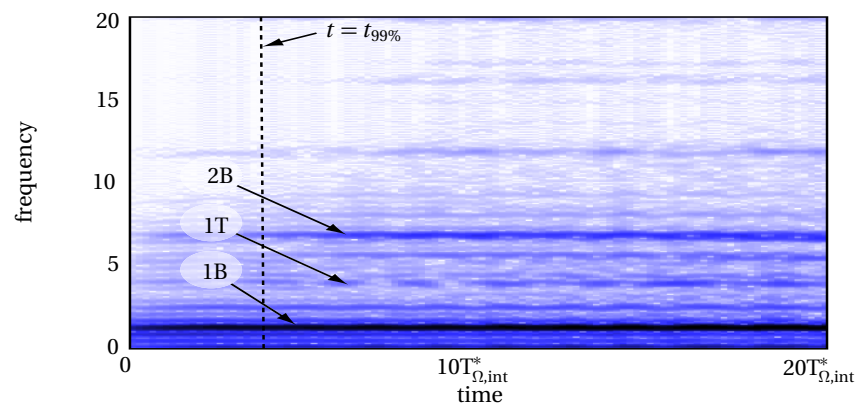

Figure 13 - Spectrogram of the tangential displacement of the contact node on the trailing edge (1B: first bending mode, 1T: first torsion mode and 2B: second bending mode of the blade).

A spectrogram of the tangential displacement of the contact node on the trailing edge is depicted in Fig. 13. The dominance of the first bending mode of the blade is underlined in agreement with experimental observations and the spectrogram in Fig. 6(b). It is noticeable that the second bending mode also has a non negligible influence over the blade dynamics.

\section{Interaction phases analysis}

For analysis purposes, backward mapping from the reduced order basis to the original physical space is performed.

\section{Phase 1}

Phase 1 is essentially characterized by a transient evolution of the casing profile. It approximatively corresponds to the first four revolutions of the blade during which the casing is ovalized. The numerical scenario features a relatively fast ovalization of the casing which is not representative of the experimental set-up. The Von Mises stress in element 1982 over the first two revolutions is pictured in Fig. 14, one may observe the increase of the mean stress level over time because of the casing ovalization.

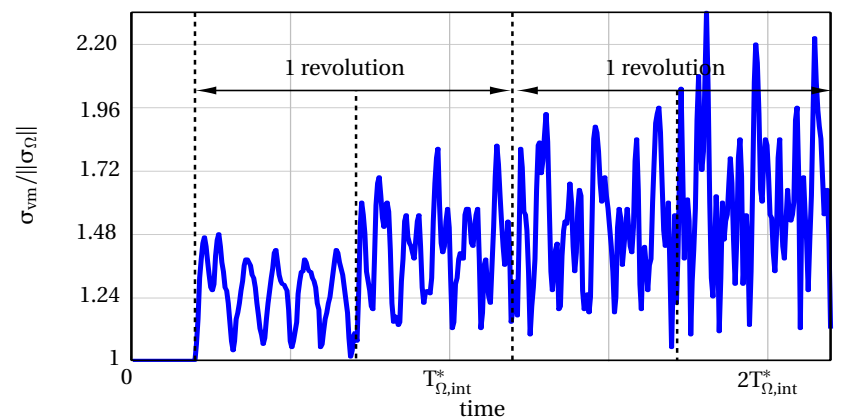

Figure 14 - Von Mises stress in element 1982 over the two first revolutions after the first bladetip/abradable contact.

The stress in the axial direction $\sigma_{\mathrm{rr}}$ in element 1982 over the second revolution is depicted in Fig. 15(a) together with its harmonic content in Fig. 15(b). It is noticeable that the mean value of $\sigma_{\mathrm{vm}} /\left\|\sigma_{\Omega}\right\|$ is about -0.9 which order of magnitude is comparable to the experimental value $\sigma_{r r, m}=$ $-1,18$ given above for the first phase of the interaction.

Finally, the harmonic content of the signal pictured in Fig. 15(b) highlights the dominance of even harmonics which shows some consistency with experimental results given in Fig. 7(b).

It was highlighted that the configuration associated with the numerical simulation features non negligible approximations (such as a perfectly ovalized casing and constant rotational velocity) with respect to the experimental set-up. However, numerical results do reveal consistency with experimental observations in terms of stress levels and harmonic content. 


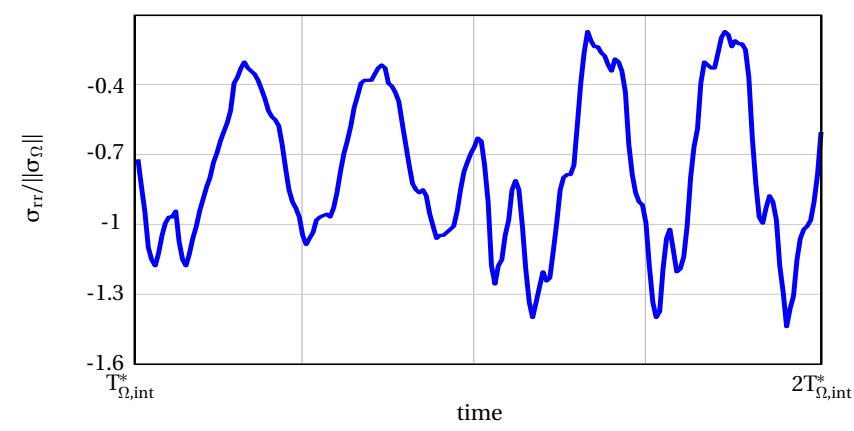

(a) Blade stress $\left(\sigma_{\mathrm{rr}}\right)$ in the middle of the blade

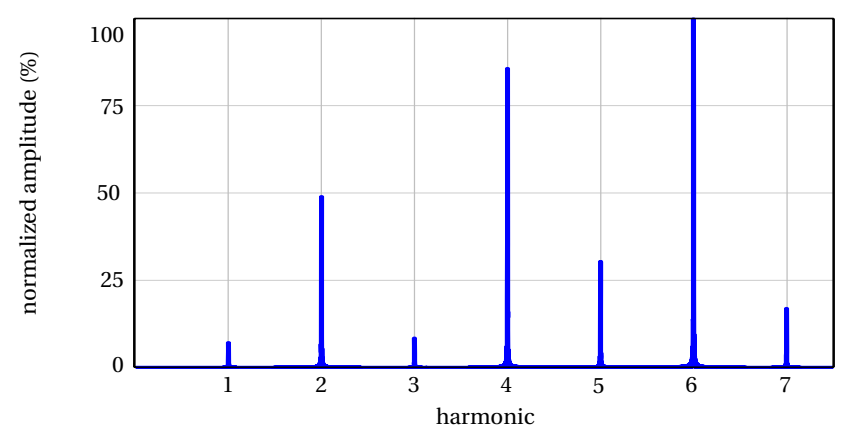

(b) Harmonic content of the blade stress signal.

Figure 15 - Blade stress $\left(\sigma_{\mathrm{rr}}\right)$ in element 1982 during the first phase of the numerical simulation (second revolution of the blade).

\section{Phase 2}

The amplitude of vibration grows linearly during the second phase as pictured in Fig. 12. Stress in the radial direction $\sigma_{\mathrm{rr}}$ in element 1982 during the eighth revolution ${ }^{4}$ of the blade is depicted in Fig. 16(a). Its average value is about -1 which is in good agreement with experimental observations during the last interaction phase in Fig. 8(a). Also, the Fourier transform of the stresses obtained experimentally and numerically - respectively given in Fig. 8(b) and in Fig. 16(b) - feature similar pattern such as the clear dominance of the sixth harmonic. This result highlight the consistency between numerical and experimental results.

Overall, these results underline a good match between the numerical simulation and experimental observations during the last interaction phase. Accordingly, it seems reasonable to use the numerical model for the observation of quantities that were not experimentally available such as the stress level in the blade root in order to understand why cracks appeared in this area.

The focus is made on the Von Mises stress within element 6455 which is visible in Fig. 10. This stress is pictured in Fig. 17(a) where it appears that the average stress value over time is close to 2.8 (about three times higher than the average radial stress in the middle of the blade). The Fourier transform of this stress plotted in Fig. 17 emphasizes the dominance of the twelfth harmonic. Frequency difference between Fig. 17 and Fig. 16(a) stems from the type of stress considered: radial stress versus Von Mises stress. Portrayed numerical evidence of possibly high cycle fatigue conditions supports the idea of crack propagation from stress concentration sites due to endurance limits [12].

A more global observation of the stress field within the blade highlights three critical areas of maximal stress levels as pictured in Fig. 18. It is remarkable that these three areas match very accurately the damaged areas experimentally observed in Fig. 5. A peak of stress occurs in the blade root.

Finally, the wear profiles obtained at the end of phase 2 are plotted in Fig. 19(a) and in Fig. 19(b) respectively for the leading edge and the trailing edge.

The resemblance of these profiles with those observed experimentally and pictured in Figs. 9(a) and 9(b) is patent. While only two main lobes are observed on the leading edge, six distinct wear lobes appear on the trailing edge. Both experimental and numerical result exhibit a higher wear level on the trailing edge in agreement with the predominance of the first bending mode in the blade dynamics. It is also noticeable that both experimental and numerical results feature four other smaller lobes over the circumference of the casing on the trailing edge so that the wear profile may be seen as a

${ }^{4}$ Similar results are observed between the sixth and the twentieth revolution. 


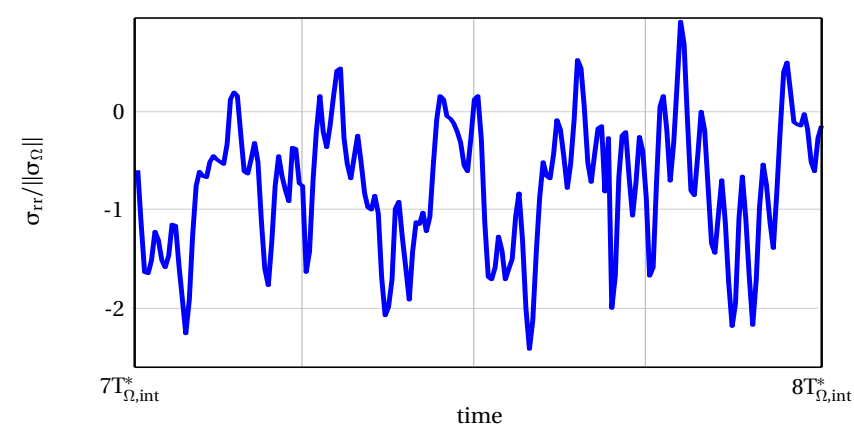

(a) $\sigma_{\mathrm{rr}}$ stress in element 1982

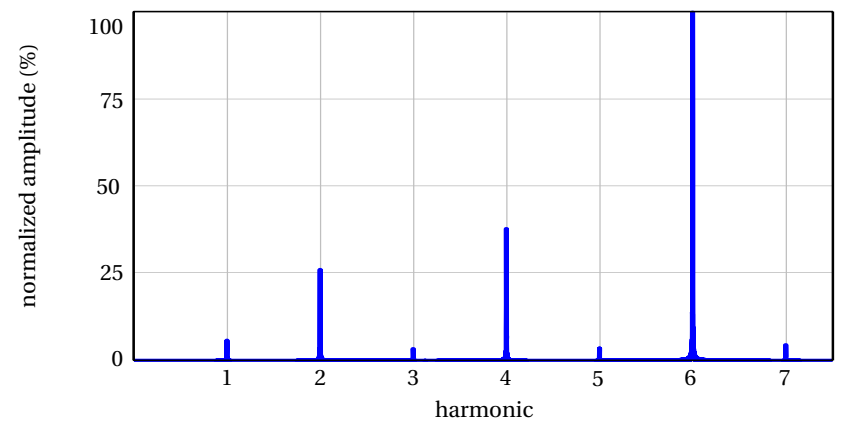

(b) Fourier transform of the $\sigma_{\mathrm{rr}}$ stress in element 1982 (assuming it periodic).

Figure 16 - Blade stress $\left(\sigma_{\mathrm{rr}}\right)$ in element 1982 during the second phase of the numerical simulation (eighth revolution of the blade).

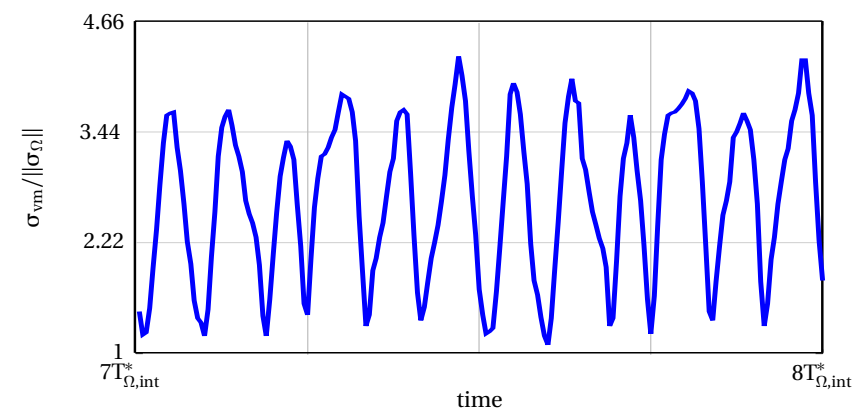

(a) Von Mises stress in element 6455

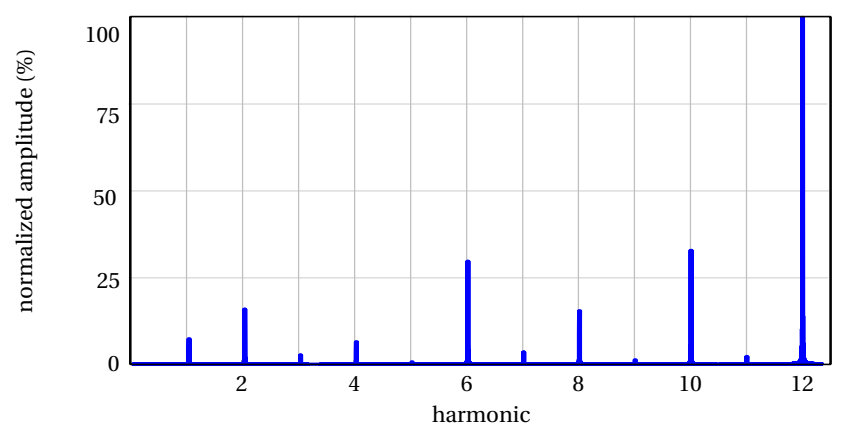

(b) Fourier transform of the Von Mises stress in element 6455 (assuming it periodic).

Figure 17 - Blade stress (Von Mises criterion) in element 6455 during the second phase of the numerical simulation (eighth revolution of the blade).

degenerated 6-lobe profile.

As previously established, a numerical tool advantageously overcomes experimental limitations in terms of instrumentation. In the following, this tool is used to carry out a numerical investigation regarding the interaction phenomenon. 


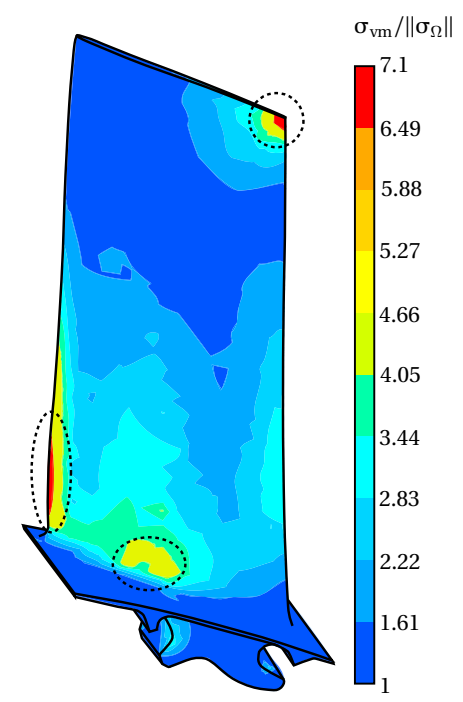

(a) revolution 8

Figure 18 - Mean predicted stress field (Von Mises criterion) inside the blade over its eigth revolution.

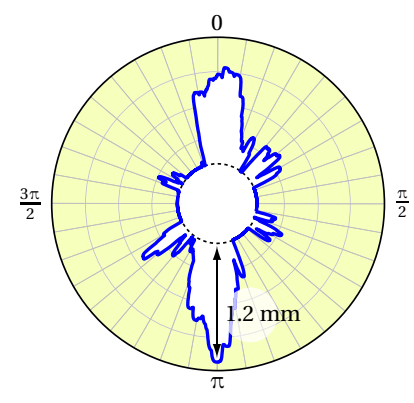

(a) leading edge

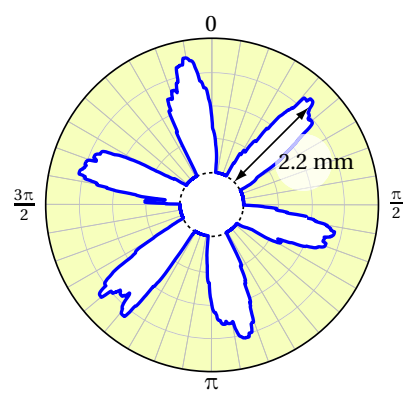

(b) trailing edge

Figure 19 - Wear of the abradable coating computed with numerical simulations.

\section{Exploratory investigation}

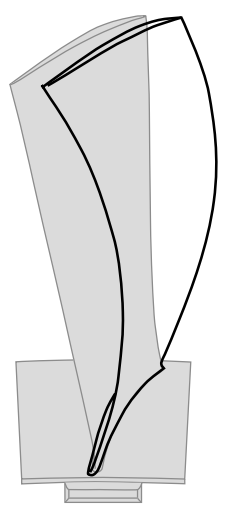

Bow

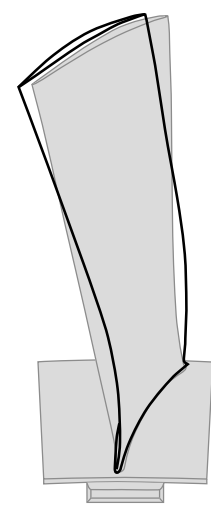

IT3

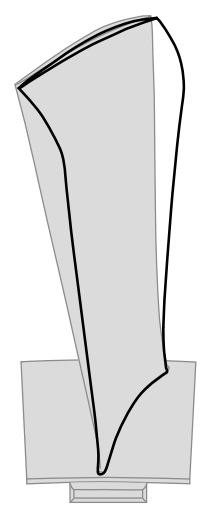

Winglet

Figure 20 - Blade design evolutions superimposed to the initial profile.

As mentioned in the first section of this paper, experimental results highlighted the fundamental role of blade dynamics in the interaction phenomenon. On that account, it seems relevant to modify the blade design in order to tackle the interaction phenomenon issue. Results presented in this section only consider a structural point of view, and do not involve any aerodynamics consideration. The three new blade profiles depicted in Fig. 20 are compared to the initial profile for a given contact configuration (ovalization of the casing) solely on their sensitivity regarding the interaction phenomenon described in the previous sections.

Fifty interaction simulations of forty revolutions of the blade inside the ovalized casing are carried 

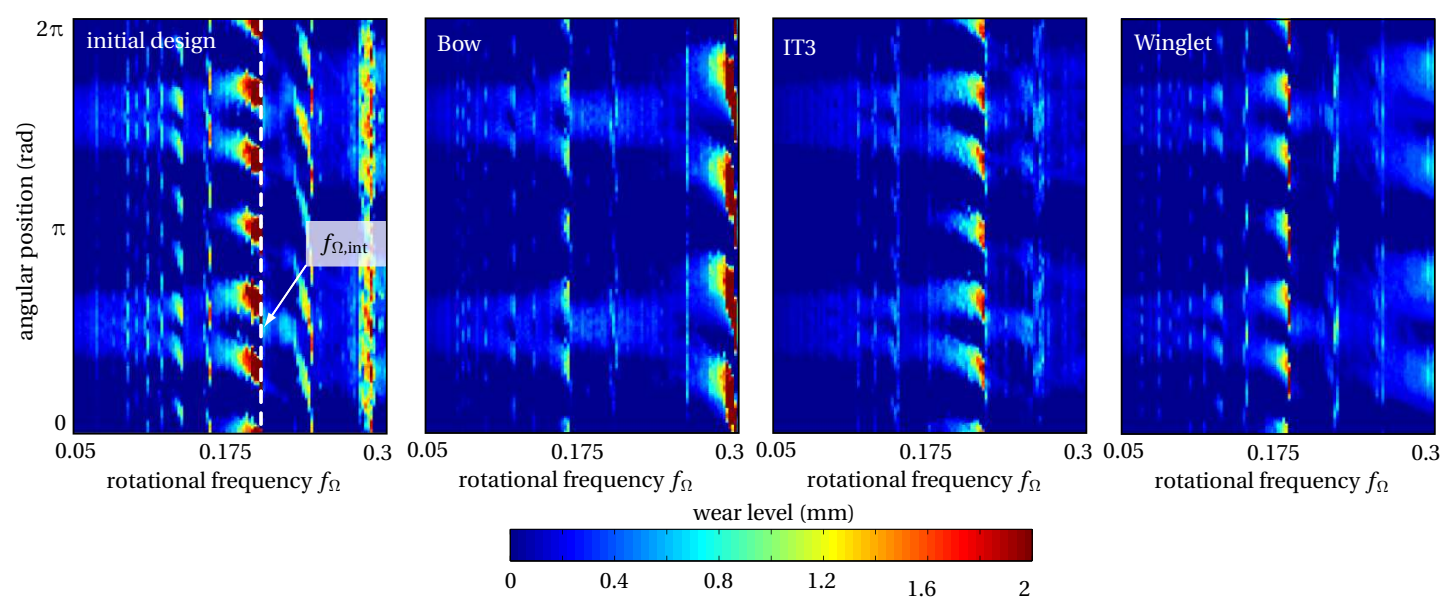

Figure 21 - Wear maps on the trailing edge for each profile of the blade (wear maps on the leading edge give similar results but are not shown here for the sake of brevity).

out over the rotational frequency range $\left[\frac{f_{\Omega \text { sint }}}{4} ; 1.6 \times f_{\Omega \text {,int }}\right]$ for each blade design. The only variable is the rotational frequency. At the end of each simulation, the wear profiles on the trailing edge are observed and put side by side so that a wear map is obtained for each blade design as pictured in Fig. 21.

The colour code is identical for the four wear maps so that the differences in terms of wear profiles appear obviously. It is remarkable that the wear level is significantly reduced when the initial design is replaced by any of the new designs. In particular, the six-lobe interaction detected for $f=f_{\Omega, \text { int }}{ }^{5}$ with the initial profile vanishes with the Bow design while it is shifted (and reduced) with the IT3 and Winglet designs.

Through this example of design evolution of the blade, it is shown that the blade-tip/abradable coating interaction phenomenon outbreak is highly dependent on the blade design.

\section{Conclusion}

This study focuses on the numerical simulation of industrial experiments where a blade-tip/ abradable coating interaction phenomenon was detected. Based on previous work related to the modeling of abradable coating and numerical developments for the analysis of modal coincidence phenomena within modern turbomachines, a numerical strategy introduced in previous publications is used for the simulation of the experiment.

While the numerically predicted interaction rotational frequency is slightly shifted with respect to the experimental one due to several assumptions (among which: rigid casing, perfectly oval shape of the casing, thermal effects neglected), numerical results are in very good agreement with experimental observations. First of all, it is highlighted that the blade dynamics is accurately captured by the numerical model since the first bending mode of the blade is dominant. Also, the numerically predicted stress levels in the middle of the blade concord with the measurements. The frequency content of the stress signal is also consistent with the experiment since it exhibits the dominance of the sixth harmonic at the end of the interaction. In terms of abradable material removal, the wear profiles measured on the casing are greatly similar with the simulated profiles: two medium lobes are observed on the leading edge while six deeper lobes are obtained on the trailing edge both experimentally and numerically. In addition, predicted critical areas in terms of stress levels within the blade match perfectly with experimental observations of the damaged blade: maximum stress areas predicted match the damaged areas of the blade at the end of the experiment.

The last part of this study is dedicated to the consequences of a design modification of the blade on its level of vibration during blade-tip/abradable coating contact simulations. The proposed designs, which are only based on structural considerations, lead to smoother vibration of the blade and no interaction speed could be found over the rotational frequency range of interest thus confirming the determinant role of blade design in the interaction phenomenon of interest.

\footnotetext{
${ }^{5}$ The natural frequencies of each blade are slightly different but remain very close. Accordingly, rotational frequencies of interest remain within $f_{\Omega} \in[0.05 ; 0.3]$.
} 


\section{Acknowledgement}

Thanks go to Snecma for its technical and financial support. This work takes place in the framework of the MAIA mechanical research and technology program sponsored by CNRS, ONERA and SAFRAN Group.

\section{Nomenclature}

$\sigma_{\Omega} \quad$ stress in the middle of the blade in the radial direction due to centrifugal effects

$\sigma_{\mathrm{Y}} \quad$ yield strength

$\sigma_{\mathrm{rr}} \quad$ stress in the radial direction

$\sigma_{\mathrm{vm}} \quad$ Von Mises stress

E Young's modulus

$f_{1} \quad$ first eigenfrequency of the blade

$f_{\Omega} \quad$ rotational frequency

$f_{\Omega \text {,int }}$ interaction rotational frequency

K Plastic modulus

$\mathrm{T}_{\Omega \text {,int }}$ interaction rotational period

\section{References}

[1] A. Millecamps, J. F. Brunel, P. Dufrénoy, F. Garcin, and M. Nucci. Influence of thermal effects during blade-casing contact experiments. In Proceedings of the ASME IDETC/CIE - DETC200986842, San Diego, USA, September 2009.

[2] P. Schmiechen. Travelling wave speed coincidence. PhD thesis, College of Science, Technology and Medicine, London, UK, 1997.

[3] A. Batailly, M. Legrand, P. Cartraud, and C. Pierre. Assessment of reduced models for the detection of modal interaction through rotor stator contacts. Journal of Sound and Vibration, 329: 5546-5562, 2010.

[4] C. Padova, J. Barton, M. Dunn, and S. Manwaring. Experimental results from controlled blade tip/shroud rubs at engine speed. Journal of Turbomachinery, 129(4):713 - 723, 2007.

[5] H.F. de Castro, K. L. Cavalca, and R. Nordmann. Whirl and whip instabilities in rotor-bearing system considering a nonlinear force model. Journal of Sound and Vibration, 317(1-2):273 - 293, 2008.

[6] R. J. Williams. Simulation of blade casing interaction phenomena in gas turbines resulting from heavy tip rubs using an implicit time marching method. In Proceedings of the ASME Turbo Expo 2011 - GT2011-45495, Vancouver, Canada, 2011.

[7] X. Ma and A. Matthews. Evaluation of abradable seal coating mechanical properties. Wear, 267: $1501-1510$.

[8] M. Yi, He J., B. Huang, and H. Zhou. Friction and wear behaviour and abradability of abradable seal coating. Wear, 231:47-53, 1999.

[9] F. Peyraut, J. L. Seichepine, C. Coddet, and M. Hertter. Finite element modeling of abradable materials - identification of plastic parameters and issues on minimum hardness against coating's thickness. International Journal for Simulation and Multidisciplinary Design Optimization, 2: 209-215.

[10] M. Legrand, A. Batailly, and C. Pierre. Numerical investigation of abradable coating removal through plastic constitutive law in aircraft engine. Journal of Computational and Nonlinear Dynamics, 7. 
[11] A. Batailly, M. Legrand, and C. Pierre. Influence of abradable coating wear mechanical properties on rotor stator interaction. In Proceedings of the ASME Turbo Expo 2011 - GT2011-45189, Vancouver, Canada, 2011.

[12] P. K. Chakravorty, V. Bhujanga Rao, K. V. V. S. S. Murthy, and T. Unnikrishnan. Vibration signature analysis of shipboard machinery. Defence Science Journal, 32(1):9-16, 1981. 